\title{
PARTHENIUM MEDIATED SYNTHESIS OF ZINC OXIDE NANOPARTICLES AND ITS CHARACTERIZATION
}

\author{
SIVA KUMAR RAMAMURTHY ${ }^{*}$, CHENCHUGARI SRIDHAR ${ }^{2}$
}

\author{
${ }^{1}$ Research scholar of Rayalaseema University, Kurnool, Andhra Pradesh, India 518007, 2(Academics), Sri Padmavathi School of Pharmacy, \\ Tiruchanoor, Andhra Pradesh, India 517503 \\ Email: sivapharma2@gmail.com
}

Received: 05 Sep 2018, Revised and Accepted: 19 Nov 2018

ABSTRACT

Objective: To biosynthesize zinc oxide nanoparticles by using parthenium hysterophorous plant extract as a reducing agent and its characterization by spectroscopic techniques.

Methods: A novel method was developed to prepare zinc oxide nanoparticles by using zinc nitrate as a precursor and biosynthesis of zinc oxide nanoparticles was mediated by parthenium hysterophorous plant extract without the aid of external energy (high pressure and temperature). This new method involves simple techniques such as centrifugation, filtration, and stirring. Zinc oxide nanoparticles formation was confirmed by analytical techniques such as UV-Visible spectroscopy, powder X-ray diffraction (XRD), Raman spectroscopy and by scanning electron microscopy (SEM) analysis.

Results: Zinc oxide nanoparticles were synthesized by using parthenium hysterophorous plant extract as a reducing agent. The XRD measurement showed that zinc oxide nanoparticles possess a typical hexagonal structure and the crystallite size of the synthesized zinc oxide nanoparticles was found to be $32 \mathrm{~nm}$ calculated by scherrer's formula. The SEM images show agglomeration of zinc oxide nanoparticles that are spherical clusters. The maximum absorbance $(380 \mathrm{~nm})$ of UV-Visible spectroscopy further confirmed synthesized nanoparticles are zinc oxide. The Raman spectra show both E2 mode and E1 mode, which indicates that the prepared zinc oxide nanoparticles possess crystalline nature with hexagonal wurtzite structure.

Conclusion: A method was established to prepare zinc oxide nanoparticles with parthenium hysterophorous plant extract which is a novel approach without the aid of external energy (high pressure and temperature), and formation of zinc oxide nanoparticles was confirmed by spectroscopic techniques. This method can be used in pharmaceutical industry for the synthesis of an antimicrobial agent.

Keywords: Zinc oxide, Nanoparticles, Parthenium hysterophorous, Raman spectra

(C) 2019 The Authors. Published by Innovare Academic Sciences Pvt Ltd. This is an open access article under the CC BY license (http://creativecommons.org/licenses/by/4.0/) DOI: http://dx.doi.org/10.22159/ijap.2019v11i1.29550

\section{INTRODUCTION}

Zinc oxide has attracted much attention due to exceptional electronic and optical properties for various technological applications, because of its wide band gap (3.2-3.7 eV) [1]. They also have remarkable potential application in the field of medicine like biological activities such as antimicrobial, antioxidant, etc.

There is a growing interest to prepare different type of nanoparticles by environmentally friendly methods that do not use toxic materials in the synthesis procedures. [2-7] Synthesis of metal oxide nanoparticles using the medicinal plant extract is quite novel, which is effective at an affordable cost, [8-12] without any external energy (high pressure, energy, temperature).

The medicinal plant parthenium hysterophorous (Feverfew) is traditionally used for vast pharmacological applications (such as treatment of fevers, migraine, headache, infertility, etc.). Among Greek and early European herbalists, the parthenium herb has a long history of use in traditional and folk medicine. Parthenium hysterophorous plant extract and zinc oxide both have antimicrobial properties. Based on the above facts, Parthenium hysterophorus plant was selected with zinc oxide metal particle for green synthesis of zinc oxide nanoparticles. The objective of the study was to establish an easy method for biosynthesis of zinc oxide nanoparticles by using parthenium hysterophorus plant extract and its characterization by spectroscopic techniques.

The rationale for the study is its pharmaceutical use as an antimicrobial agent. Easy method for synthesis without the aid of external energy such as high pressure and temperature. This study is a novel approach that describes the easy synthesis of zinc oxide nanoparticles without heat treatment, using parthenium hysterophorus plant extract. These zinc oxide nanoparticles were characterized by spectroscopic techniques for the confirmation of the formation of zinc oxide nanoparticles. This research study provides an established method for biosynthesis of zinc oxide nanoparticles which can be used as an antimicrobial agent in the pharmaceutical industry.

\section{MATERIALS AND METHODS}

\section{Parthenium hysterophorus plant material}

Flowers and leaves of parthenium hysterophorus plant were collected from Indira park and public gardens, Nampally, Hyderabad. Parthenium hysterophorus plant of family Asteraceae was identified by the Department of Botany, Sri Venkateswara University, Tirupati with voucher number 1216. The plant was identified based on the leaves, lobed with fine soft hair, flowers on the top are small creamy colored with black colored seed. Based on the features of the plant it was confirmed as parthenium hysterophorus.

\section{Preparation of parthenium hysterophorus plant extract}

After the identification of the plant, the leaves and flowers were separated from the plant. The leaves and flowers were dried under dark and shady conditions, without exposing the material to sunlight. After drying, leaves and flowers were powdered in a mechanical grinder, and the fine powder was collected by passing through sieve no 40 . This powder is stored in a cool and dry place until its use. Plant powder was extracted in a number of solvents such as methanol, hexane, anhydrous sodium sulfate, acetone, chloroform, diethyl ether. Of all the solvents used, acetone is considered as the best solvent for the extraction of the compound from the leaves and flowers of parthenium hysterophorus plant.

$50 \mathrm{~g}$ of powdered parthenium hysterophorous plant material was weighed and carefully transferred into the round-bottomed flask of Soxhlet extractor. Then $250 \mathrm{ml}$ of acetone was added, and the plant 
material was soaked in acetone for $24 \mathrm{~h}$ at room temperature. Then the acetone extract of the plant was filtered using Whatman no 1 filter paper. This supernatant is taken in a separate beaker. This crude extract was used only for further analysis.

\section{Biosynthesis of zinc oxide nanoparticles with parthenium hysterophorous plant extract}

A quantity of $1 \mathrm{~g}$ of parthenium hysterophorous plant extract was dissolved in $100 \mathrm{ml}$ of de-ionized water and centrifuged for $15 \mathrm{~min}$ and filtered. Zinc nitrate $0.75 \mathrm{~g}(0,1 \mathrm{M})$ was used as the precursor for the preparation of zinc oxide nanoparticles. $40 \mathrm{ml}$ of the extract of parthenium hysterophorous was added dropwise in zinc precursor while stirring using a magnetic stirrer. In order to adjust the $\mathrm{pH}=12$ of the solution, sodium hydroxide $(\mathrm{NaOH}, 1 \mathrm{M})$ was added drop-wise while stirring. A white crystalline precipitate of zinc oxide was obtained, which is washed 2-3 times with de-ionized water, filtered and dried to obtain the zinc oxide nanoparticles.

\section{RESULTS AND DISCUSSION}

\section{Characterization of zinc oxide nanoparticles}

\section{a. Powder X-ray diffraction}

XRD was taken to examine the crystal structure and phase purity of synthesized zinc oxide nanoparticles using the extract of parthenium hysterophorous plant without annealing. Fig. 1 shows the corresponding XRD pattern and below bars are the hexagonal phase. As can be seen from the fig. of the obtained XRD pattern consists of dominant peaks are consistent with the zinc oxide hexagonal phase (standard joint committee on powder diffraction standards (JCPDS) card no. 36-1451) [13]. The parthenium hysterophorous plant extracts contains phytochemicals helps in the synthesis of metal oxide nanoparticle by inducing oxidation and reduction reaction. Further, the pattern shows a line broadening, which indicates the crystallite size reduced.

The crystallite size of the material was calculated using the Scherrer's formula:

$$
\delta=\mathrm{K} \lambda / \beta \chi \mathrm{o} \sigma \theta,
$$

Where $d$ is the crystallite size, $\mathrm{K}$ is the dimensionless shape factor (0.94), $\lambda$ is the X-ray wavelength, $\beta$ is the full-width half maxima (FWHM) and $\square$ is the Bragg's angle. The crystallite size of the synthesized $\mathrm{ZnO}$ was found to be $32 \mathrm{~nm}$.

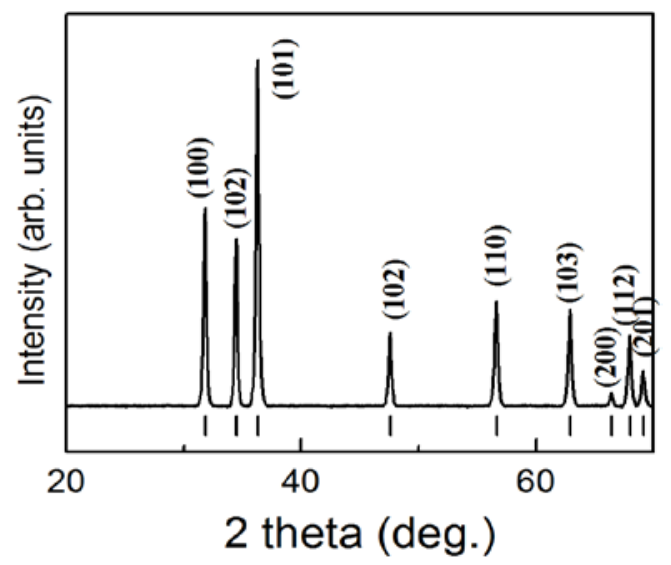

Fig. 1: Room temperature powder XRD pattern of as-prepared zinc oxide nanoparticles using parthenium extract

\section{b. Scanning electron microscopy}

The SEM images show agglomeration of zinc oxide particles (fig. 2(a)). The magnified image is shown in fig. 2(b), it appears to be some of the particles are spherical. Fig. 2 represents the morphology of the assynthesized zinc oxide nanoparticles prepared by using parthenium hysterophorous plant extract. Typical SEM images of the zinc oxide nanostructures at two different magnifications are shown in fig. 2(a) and $(b)$. It is clear from the lower magnification image that the assynthesized zinc oxide nanoparticles are spherical clusters in a largescale area and have approximately uniform morphology. Fig. 2(b) shows the higher magnification image of such spherical particles surrounded by amorphous parthenium hysterophorous plant extract.
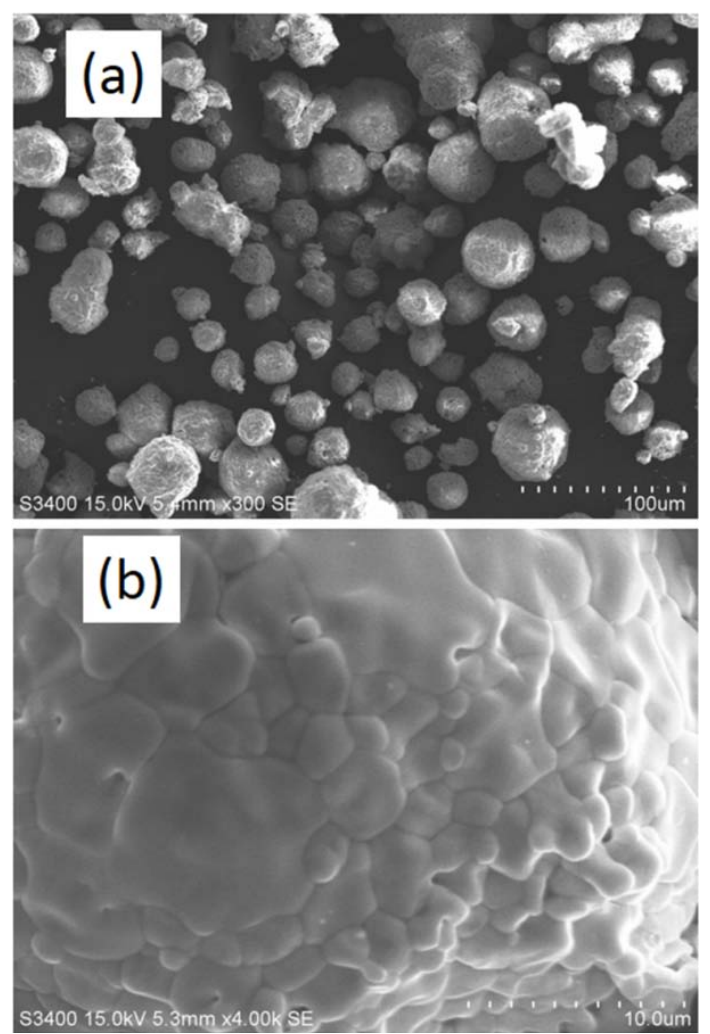

Fig. 2: SEM images of as-prepared zinc oxide nanoparticles using parthenium extract at different magnitudes (a) $100 \square \mathrm{m}$ and (b) $10 \mu \mathrm{m}$

\section{c. UV-visible spectroscopy}

UV-visible absorption spectrum as showed in fig. 3 , is carried out to evaluate the potential optical properties of the as-prepared zinc oxide nanoparticles using parthenium hysterophorous plant extract. For the UV-visible absorption measurement, the as-prepared zinc oxide nanoparticles using parthenium hysterophorous plant extract sample is ultrasonically dispersed in absolute ethanol before the examination, using absolute ethanol as the reference. The spectrum was corrected for the solvent contribution. The absorption spectrum of zinc oxide nanoparticles using parthenium hysterophorous plant extract shows well-defined excitation band at $\sim 401.5 \mathrm{~nm}$, which is shifted by $\sim 28 \mathrm{~nm}$ relative to the bulk zinc oxide excitation absorption [14] higher than that of the bulk zinc oxide excitation band $(\sim 373.5 \mathrm{~nm})$. The calculated band gap of $\sim 3.09 \mathrm{eV}$ of these nanoparticles is less than that of the band gap of bulk zinc oxide $(3.3$ $\mathrm{eV}$ ). The reason for the shifting of the absorption band could be due to the oriented attachment of the nanoparticles by using parthenium extract may lead to defect formation in these zinc oxide nanoparticles. Similar observations for shifting of absorption bands of zinc oxide towards the visible region were also reported earlier [15]. Surface area and surface defects play an important role in the photocatalytic activities of metal oxides. Additionally, it affects the optical and electronic properties due to which the optical absorption shifts towards the visible region. For the effective use of zinc oxide, band gap has to be minimized from $3.38 \mathrm{eV}$ to below $2.0 \mathrm{eV}$, since it is the recommended band gap value for achieving a visible-light active photocatalyst [16].

However, pure zinc oxide phase acts as an efficient photo catalyst only under UV irradiation. One of the strategies adopted for tuning 
the band gap is to introduce intentional defects in the crystal lattice in which the electronic structure of zinc oxide can be altered.

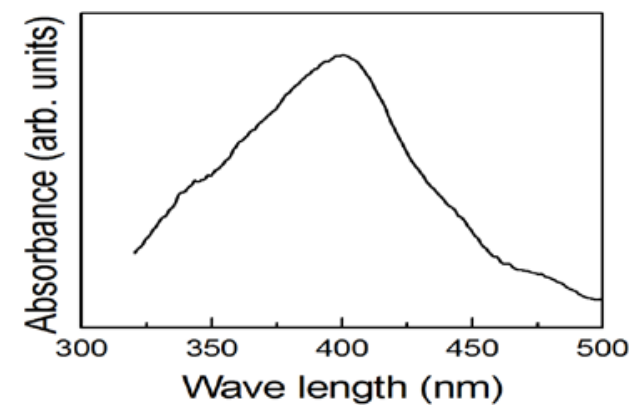

Fig. 3: Absorption of as-prepared zinc oxide nanoparticles using parthenium extract as a function of wavelength

\section{d. Raman spectroscopy}

The Raman spectrum is an important and useful tool to investigate the optical, vibrational properties of materials and to identify the crystallization, structural disorder, and material defects. According to group theory, the optical phonons at the $\Gamma$ point of Brillouin zone are $1 \mathrm{~A}_{1}+2 \mathrm{~B}_{1}+1 \mathrm{E}_{1}+2 \mathrm{E}_{2}$ for hexagonal wurtzite zinc oxide. Among them, both A1 and E1 models are polar and split into transverse (TO) and longitudinal optical (LO) photons, all being Raman and infrared active. The two nonpolar E2 modes are Raman active only and the two B1 modes are infrared. Furthermore, nonpolar E2 photon modes have two frequencies. The low-frequency E2 mode (E2 low) is associated with vibration of zinc (Zn) sublattice. Whereas the high-frequency E2 mode (E2 high) is related to the vibration of oxygen atoms [17-19]. The frequencies of the fundamental optical modes in zinc oxide reported in the literature [20] are: E2 low = 101 $\mathrm{cm}^{-1}$, E2 high $=437 \mathrm{~cm}^{-1}, \mathrm{~A} 1(\mathrm{TO})=380 \mathrm{~cm}^{-1}, \mathrm{~A} 1(\mathrm{LO})=574 \mathrm{~cm}^{-1}, \mathrm{E} 1$ (TO) $=407 \mathrm{~cm}^{-1}$, E1 (LO) $=583 \mathrm{~cm}^{-1}$, E2 high-E2 low $=333 \mathrm{~cm}^{-1}$. The observed room temperature Raman spectrum of as-prepared zinc oxide nanoparticles using parthenium plant extract exhibits multiple prominent peaks in addition to weak and broad peaks as showed in fig. 4. The observed spectrum shows an intense peak at $439 \mathrm{~cm}^{-1}$, which corresponds to the Raman active E2 (high) mode of the wurtzite zinc oxide crystal. It is red shifted of $\sim 2 \mathrm{~cm}^{-1}$ as compared with the bulk zinc oxide. The second significant Raman active peak at $342 \mathrm{~cm}^{-1}$ could be assigned to the second order Raman spectrum arising from zone-boundary phonons E2 high-E2 low, while the small peak at $398 \mathrm{~cm}-1$ corresponds to A1 (TO) phonon modes and found to be redshifted at $18 \mathrm{~cm}^{-1}$. The small peak at $589 \mathrm{~cm}-1$ is contributed by the E1 (LO) mode of as-prepared zinc oxide nanoparticles using parthenium plant extract associated with the formation of defects such as oxygen vacancies or other defect states. The observed intense E2 (high) mode and suppressed E1 (LO) mode indicates that the as-prepared zinc oxide nanoparticles using parthenium plant extracts possess crystalline nature with hexagonal wurtzite structure, which further testifies the results of XRD pattern.

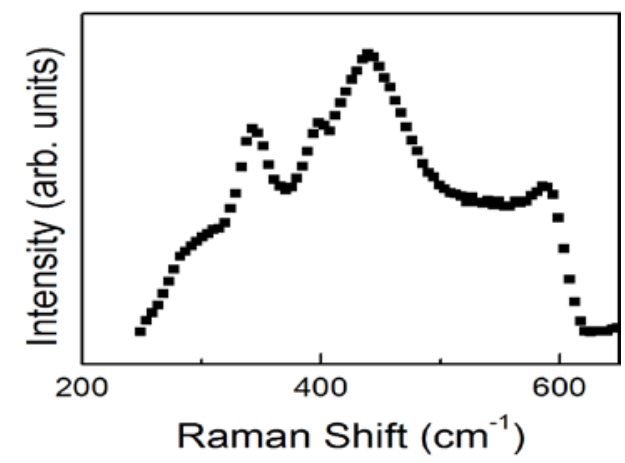

Fig. 4: Raman spectra of as-prepared zinc oxide nanoparticles using parthenium plant extract

\section{CONCLUSION}

A novel eco-friendly method was established to synthesize Zinc oxide nanoparticles without the aid of external energy. Formation of these nanoparticles was confirmed by spectroscopic techniques. Zinc oxide nanoparticles is a proven antimicrobial agent with minimal effect on human cells. Hence the established method can be further scaled up for zinc oxide nanoparticle synthesis for pharmaceutical use.

\section{ACKNOWLEDGMENT}

The Author is very much thankful to Mysore University, Mysore for facilitating the analytical services. The Author is thankful to his mentor for his continuous support and guidance.

\section{AUTHORS CONTRIBUTIONS}

All the authors have contributed equally

\section{CONFLICTS OF INTERESTS}

\section{Declared none}

\section{REFERENCES}

1. SJ Pearton, DP Norton, K Ip, YW Heo. Recent advances in the processing of ZnO. J Vacuum Sci Technol B: Microelectronics Nanometer Structures Processing Measurement Phenomena 2004;22:932-48.

2. Poovathinthodiyil Raveendran, Jie $\mathrm{Fu}$, Scott $\mathrm{L}$ Wallen. Completely "Green" synthesis and stabilization of metal nanoparticles. J Am Chem Soc 2003;125:13940-1.

3. Narayanan Sreeja, Binulal Nelson Sathy, Ullas Mony, Manzoor Koyakutty, Shantikumar Vasudevan Nair, Deepthy Menon. Biocompatible magnetite/gold nanohybrid contrast agents via green chemistry for MRI and CT bioimaging. ACS Appl Mater Interfaces 2012;41:251-60.

4. Anju Thangam, Sakthi Ramlakshmi, Pritam. Effect of $\mathrm{ZnO}$ nanoparticles against strains of Escherichia coli. Asian J Pharm Clin Res 2014; 7:202-6.

5. Subramani Srinivasan, Dhananjayan Indumathi, Mathiyazhagan Sujatha, Kathiroli Sujithra, Udaiyar Muruganathan. Novel synthesis, characterization and antibacterial activity of silver nanoparticles using leaf extract of Melothria maderaspatana (linn) cong. Int J Pharm Pharm Sci 2016;8:104-9.

6. Pramod Kumar, Indrajit Roy. Applications of gold nanoparticles in clinical medicine. Int J Pharm Pharm Sci 2016;8:9-16.

7. Nachiyar V, Sunkar S, Prakash P. Biological synthesis of gold nanoparticles using endophytic fungi. Der Pharm Chem 2015;7:31-8.

8. Ramesh M, Anbuvannan M, Viruthagiri G. Green synthesis of $\mathrm{ZnO}$ nanoparticles using Solanum nigrum leaf extract and their antibacterial activity. Spectrochim Acta Part A 2015;136:864-70.

9. Xiao L, Liu C, Chen X, Yang Z. Zinc oxide nanoparticles induce renal toxicity through reactive oxygen species. Food Chem Toxicol 2016;90:76-83.

10. Rajeshkumar S. Anticancer activity of eco-friendly gold nanoparticles against lung and liver cancer cells. J Genetic Eng Biotechnol 2016;14:195-202.

11. C Nagajyothi P, An Tran Nguyen Tvm. Green route biosynthesis: characterization and catalytic activity of $\mathrm{ZnO}$ nanoparticles. Materials Lett 2013;108:160-3.

12. Mahendran Vanaja, Gnanadhas Gnanajobitha, Kanniah Paulkumar, Shanmugam Rajeshkumar, Chelladurai Malarkodi, Gurusamy Annadurai. Phytosynthesis of silver nanoparticles by Cissus quadrangularis: Influence of physicochemical factors. J Nanostruct Chem 2013;3:17-24.

13. HF McMurdie, MC Morris, EH Evans, B Paretzkin, W Wong-Ng, L Ettlinger, et al. Standard X-ray diffraction powder patterns from the JCPDS research associateship. Powder Diffr 1986;1:64-77.

14. Markus Haase, Horst Weller, Arnim Henglein. Photochemistry and radiation chemistry of colloidal semiconductors. 23. Electron storage on zinc oxide particles and size quantization. J Physical Chem 1988;92:482-7.

15. Lili Wu, Youshi $\mathrm{Wu}, \mathrm{Wei}$ LU. Preparation of $\mathrm{ZnO}$ Nanorods and optical characterizations. Physica E 2005;28:76-82. 
16. Sunandan Baruah, Sudarson Sekhar Sinha, Barnali Ghosh, Samir Kumar Pal, AK Raychaudhuri, Joydeep Dutta. Photoreactivity of $\mathrm{ZnO}$ nanoparticles in visible light: effect of surface states on electron transfer reaction.J Appl Physics 2009;105:074308-1.

17. A Arguello C, Rousseau, Denis, Porto, Simone. First-order raman effect in wurtzite-type crystals. Phys Rev J 1969 181:1351-63.
18. Lin KF, Cheng HM, Hsu HC, Hsieh WF. Band gap engineering and spatial confinement of optical phonon in $\mathrm{ZnO}$ quantum dots. Appl Phys Lett 2006;88:263117-9.

19. Khan A Alim, Vladimir A Fonoberov, Manu Shamsa, Alexander A Balandin. Micro-Raman investigation of optical phonons in ZnO nanocrystals. J Appl Phys 2005;97:124313-7.

20. TC Damen, SPS Porto, B Tell. Raman effect in zinc oxide. Phys Rev J 1966;142:570-4. 